\title{
Correction to: Reduced nNOS activity is responsible for impaired fatty acid-dependent mitochondrial oxygen consumption in atrial myocardium from hypertensive rat
}

\author{
Yu Na Wu ${ }^{1,2}$. Chun Li Jin ${ }^{1}$ • Ji Hyun Jang ${ }^{3}$ - Zai Hao Zhao ${ }^{1}$. Sung Joon Kim ${ }^{2}$ • Yin Hua Zhang ${ }^{1,2,4}$ \\ Published online: 23 July 2020 \\ (C) Springer-Verlag GmbH Germany, part of Springer Nature 2020
}

Correction to: Pflügers Archiv - European Journal of Physiology (2020)

https://doi.org/10.1007/s00424-020-02435-9

The above article was published online with an error in affiliations. This is the correct affiliations of the authors. "Yu Na $\mathrm{Wu}^{1,2}$, Chun Li Jin ${ }^{1}$, Ji Hyun Jang ${ }^{3}$, Zai Hao Zhao ${ }^{1}$, Sung Joon $\mathrm{Kim}^{2}$, Yin Hua Zhang ${ }^{1,2,4}$. The correct affiliations is also presented above.

The online version of the original article can be found at https://doi.org/ $10.1007 / \mathrm{s} 00424-020-02435-9$

\footnotetext{
Yin Hua Zhang

yinzhang@snu.ac.kr

1 Yanbian University Hospital, Yanbian University, Yanji 133000,

China

2 Department of Physiology \& Biomedical Sciences, Ischemic/ Hypoxic Disease Institutes, Seoul National University, College of Medicine, Seoul 03080, Republic of Korea

3 University of Maryland School of Medicine, Baltimore, USA

4 Institute of Cardiovascular Sciences, Faculty of Biology, Medicine and Health Sciences, University of Manchester, Manchester, UK
} 